\title{
THE NONEQUIVALENCE OF OSCILLATION AND NONDISCONJUGACY
}

\author{
G. B. GUSTAFSON
}

Abstract. A sufficient condition is given for all solutions of the adjoint of an $n$th order linear differential equation to have an infinity of zeros; an example is presented which shows that for every integer $n>2$, there exists an $n$th order equation, all of whose solutions have a finite number of zeros, but the adjoint has only solutions with an infinity of zeros. In addition, some open equations on conjugate points are answered.

1. Introduction. The adjoint equation for the $n$th order linear differential equation

$$
x^{(n)}+\sum_{k=0}^{n-1} P_{k}(t) x^{(k)}=0
$$

is defined to be the equation (see $[5$, p. 66])

$$
x^{(n)}+\sum_{k=0}^{n-1}(-1)^{n-k}\left(p_{k}(t) x\right)^{(k)}=0 .
$$

Zeros of nontrivial solutions to such equations are counted according to multiplicities (see [5, p. 67]). The nth conjugate point to $a$ is defined as in [1] and [3], and is denoted by $\eta_{p}(a)$ whenever it exists. Nontrivial solutions are oscillatory if they have an infinity of zeros, and are nonoscillatory, otherwise; if all solutions are oscillatory, then the equation is said to be strongly oscillatory [2]. Nonoscillatory equations have all solutions nonoscillatory, and otherwise the equation is oscillatory. Disconjugate on a connected set $E$ means that the number of zeros of a nontrivial solution cannot equal the order of the equation.

In personal conversations with the late Professor J. L. Barrett, and Professor T. L. Sherman, the following open questions were formulated for higher order equations:

(i) Does the existence of $\eta_{P}(t)$ for all $t$, all $p \geqq 1$, imply oscillation?

(ii) Does nondisconjugacy on every half-line imply oscillation?

(iii) Does oscillation imply oscillation of the adjoint?

(iv) Does strong oscillation imply oscillation of the adjoint?

Received by the editors August 11, 1969.

A MS Subject Classifications. Primary 3442; Secondary 3430, 3450.

Key Words and Phrases. $n$th conjugate point, oscillatory, nonoscillatory, strongly oscillatory, disconjugate, zeros counted according to multiplicity. 
The purpose of this paper is to answer (i)-(iv) in the negative for $n>2$. Classes of equations for which (i)-(iv) are true have been investigated by Hanan [4].

2. Lemma. If $f$ and $g$ belong to $C^{2}[0, \infty)$ and $f^{2} g^{\prime}>0$, then $y_{1}(t)$ $=f(t) \cos g(t)$ and $y_{2}(t)=f(t) \sin g(t)$ form a fundamental set of solutions for the differential equation $\left(p(t) y^{\prime}\right)+q(t) y=0$, where $p=1 /\left(f^{2} g^{\prime}\right)$ and

$$
q=\frac{g^{\prime}}{f^{2}}-\frac{1}{f}\left[\frac{f^{\prime}}{f^{2} g^{\prime}}\right]^{\prime} .
$$

3. Lemma. Suppose $x(t)$ and $y(t)$ are linearly independent solutions of the equation $\left(p(t) y^{\prime}\right)^{\prime}+q(t) y=0$. The general solutions of $\left[\left(p(t) z^{\prime}\right)^{\prime}+q(t) z\right]^{(n)}=0$ and $\left(p(t) w^{(n+1)}\right)^{\prime}+q(t) w^{(n)}=0$ have the form

$$
\begin{aligned}
& z(t)=a x(t)+b y(t)+\sum_{k=0}^{n-1} C_{k} \int_{0}^{t}[x(t) y(s)-x(s) y(t)] s^{k} d s, \\
& w(t)=\sum_{k=0}^{n-1} C_{k} t^{k}+a \int_{0}^{t} \frac{(t-s)^{n-1}}{(n-1) !} x(s) d s+b \int_{0}^{t} \frac{(t-s)^{n-1}}{(n-1) !} y(s) d s,
\end{aligned}
$$

respectively, where $a, b, C_{0}, C_{1}, \cdots, C_{n-1}$ are arbitrary constants.

Throughout the remainder of the paper, $L z=0$ shall denote the equation $\left[\left(p(t) z^{\prime}\right)^{\prime}+q(t) z\right]^{(n)}=0$ and $L^{*} w=0$ shall denote $\left(p(t) w^{(n+1)}\right)^{\prime}$ $+q(t) w^{(n)}=0$. If $p(t)$ and $q(t)$ are sufficiently differentiable, then the adjoint of $L z=0$ is the product of the adjoint of $\left(p(t) z^{\prime}\right)^{\prime}+q(t) z=0$ with the adjoint of $y^{(n)}=0$, and both $L z=0$ and its adjoint have the form (1.1). In particular, if $p(t)$ and $q(t)$ are of class $C^{n+1}$, then $L^{*} w=0$ is the adjoint of $L z=0$, and both have the form (1.1).

4. Lemma. Let $F(t), G(t), H(t)$ be continuous on $E=[a, b)$, and suppose that $G(t)$ and $H(t)$ are positive. If the Lebesgue integrals $\int_{E} F(t) G(t) d t$, $\int_{E} F(t) H(t) d t$ equal $+\infty$ and $\lim _{t \rightarrow b} G(t) / H(t)=A$, then

$$
\lim _{t \rightarrow b} \frac{\int_{a}^{t} F(s) G(s) d s}{\int_{a}^{t} F(s) H(s) d s}=A .
$$

5. Lemma. Suppose $x(t)$ and $y(t)$ are linearly independent solutions of the equation $\left(p(t) z^{\prime}\right)^{\prime}+q(t) z=0$, and $p(t)$ and $q(t)$ belong to $C^{n+2}[0,+\infty)$. If

$$
\int_{0}^{\infty} y(s) s^{k} d s=+\infty \quad(0 \leqq k \leqq n-1),
$$


(ii) $\lim _{t \rightarrow \infty} \int_{0}^{t} x(s) d s=0$ and $\int_{0}^{t} x(s) d s>0 \quad$ for $t>0$,

then all nontrivial solutions of $L z=0$ are oscillatory and all nontrivial solutions of $L^{*} w=0$ are nonoscillatory.

Proof. It will be demonstrated that all nontrivial solutions of $L z=0$ have an infinity of zeros on $[0,+\infty)$. By Lemma 3 , a nontrivial solution of $L z=0$ has the form

$$
z(t)=x(t)\left[a+\sum_{k=0}^{n-1} C_{k} \int_{0}^{t} y(s) s^{k} d s\right]+y(t)\left[b-\sum_{k=0}^{n-1} C_{k} \int_{0}^{t} x(s) s^{k} d s\right],
$$

where at least one of the constants $a, b, C_{0}, \cdots, C_{n-1}$ is not zero. To prove that $z(t)$ is oscillatory, it will be shown that $z(t)$ changes sign on every half-line. There is nothing to prove if all the $C_{k}$ 's are zero. Otherwise, there is a largest index $j$ such that $C_{j} \neq 0$. By (i) of the hypothesis, and Lemma 4 ,

$$
\lim _{t \rightarrow \infty} \frac{a+\sum_{k=0}^{n-1} C_{k} \int_{0}^{t} y(s) s^{k} d s}{\int_{0}^{t} y(s) s^{j} d s}=C_{j},
$$

and therefore $a+\sum_{\substack{n=0 \\ n-1}} C_{k} \int_{0}^{t} y(s) s^{k} d s$ is nonzero on some half-line $[T,+\infty)$. Given any half-line, $y(t)$ has two consecutive zeros $t_{0}, t_{1}>T$ in this half-line. The Wronskian of $x(t)$ and $y(t)$ equals $c / p(t)$ for some nonzero constant $c$. Since

$$
z\left(t_{i}\right)=\frac{c}{y^{\prime}\left(t_{i}\right) p\left(t_{i}\right)}\left[a+\sum_{k=0}^{n-1} C_{k} \int_{0}^{t_{i}} y(s) s^{k} d s\right], \quad i=0,1,
$$

and $y^{\prime}(t)$ has different signs at $t_{0}$ and $t_{1}$, it follows that $z(t)$ must change sign between $t_{0}$ and $t_{1}$. Therefore, $z(t)$ is oscillatory.

To prove that $L^{*} w=0$ is nonoscillatory, let $w(t)$ be a nontrivial solution of this equation, and suppose that $w(t)$ has infinitely many zeros on $t \geqq 0$, in order to obtain a contradiction.

Since all derivatives of $w(t)$ must vanish infinitely of ten on every half-line, Lemma 3 says every half-line contains points $t$ such that

$$
(n-1) ! C_{n-1}+a \int_{0}^{t} x(s) d s+b \int_{0}^{t} y(s) d s=0 .
$$

Since by hypothesis $\lim _{t \rightarrow \infty} \int_{0}^{t} x(s) d s=0$ and $\lim _{t \rightarrow \infty} \int_{0}^{t} y(s) d s=\infty$, it 
follows that $b=0$. But then $C_{n-1}=0$, and since $\int_{0}^{t} x(s) d s>0$ for $t>0$, $a=0$ also. Therefore, $w(t)$ must be a nonzero polynomial with an infinity of zeros; the contradiction is reached, and the proof is complete.

6. Lemma. If $x(t)$ and $y(t)$ belong to $C[0, \infty)$ and are linearly independent solutions of $w^{\prime \prime}+k^{2} w=0$ throughout an interval $c \leqq t \leqq d$, $c \geqq 0$, then for each number $a, 0 \leqq a \leqq c$, there exists a polynomial $Q(t)$ $=a_{n-1} t^{n-1}+\cdots+a_{1} t+a_{0}$ and constants $C_{1}$ and $C_{2}$ such that

$$
w(t)=C_{1} \int_{0}^{t} \frac{(t-s)^{n-1}}{(n-1) !} x(s) d s+C_{2} \int_{0}^{t} \frac{(t-s)^{n-1}}{(n-1) !} y(s) d s+Q(t)
$$

vanishes at $t=a$ and is a nontrivial solution of $w^{\prime \prime}+k^{2} w=0$ on $c \leqq t \leqq d$.

Proof. The Taylor formula

$$
F(t)=\sum_{k=0}^{n-1} \frac{F^{(k)}(c)}{k !}(t-c)^{k}+\int_{c}^{t} \frac{(t-s)^{n-1}}{(n-1) !} F^{(n)}(s) d s
$$

can be applied to $F(t)=x(t), y(t)$ on $c \leqq t \leqq d$ to get

$$
\begin{aligned}
& \int_{c}^{t} \frac{(t-s)^{n-1}}{(n-1) !} x(s) d s=Q_{1}(t)+b_{1} \cos \left(k t+\theta_{1}\right), \\
& \int_{c}^{t} \frac{(t-s)^{n-1}}{(n-1) !} y(s) d s=Q_{2}(t)+b_{2} \cos \left(k t+\theta_{2}\right),
\end{aligned}
$$

where $Q_{1}(t)$ and $Q_{2}(t)$ are polynomials of degree at most $n-1, b_{1} b_{2}$ $\neq 0, \theta_{1} \neq \theta_{2}$ modulo $\pi$.

Select constants $C_{1}$ and $C_{2}$ not both zero such that

$$
\begin{aligned}
C_{1}\left[Q_{1}(a)+\int_{a}^{e} \frac{(a-s)^{n-1}}{(n-1) !}\right. & x(s) d s] \\
& +C_{2}\left[Q_{2}(a)+\int_{a}^{c} \frac{(a-s)^{n-1}}{(n-1) !} y(s) d s\right]=0 .
\end{aligned}
$$

Put

$$
\begin{aligned}
Q(t)= & -C_{1} \int_{0}^{c} \frac{(t-s)^{n-1}}{(n-1) !} x(s) d s-C_{2} \int_{0}^{c} \frac{(t-s)^{n-1}}{(n-1) !} y(s) d s \\
& -C_{1} Q_{1}(t)-C_{2} Q_{2}(t) .
\end{aligned}
$$

Then $Q(t)$ has the form asserted and

$$
w(t)=C_{1} \int_{0}^{t} \frac{(t-s)^{n-1}}{(n-1) !} x(s) d s+C_{2} \int_{0}^{t} \frac{(t-s)^{n-1}}{(n-1) !} y(s) d s+Q(t)
$$


vanishes at $t=a$. On the interval $c \leqq t \leqq d$,

$$
w(t)=C_{1} b_{1} \cos \left(k t+\theta_{1}\right)+C_{2} b_{2} \cos \left(k t+\theta_{2}\right) .
$$

Since $b_{1} b_{2} \neq 0, \theta_{1} \neq \theta_{2}$ modulo $\pi$, and not both $C_{1}$ and $C_{2}$ are zero, $w(t)$ is a nontrivial solution of $w^{\prime \prime}+k^{2} w=0$ on $c \leqq t \leqq d$.

A proof of the next lemma can be found in [3].

7. Lemma. For every integer $N>0$, there exists an $r(t)$ in $C^{N}[0,1]$ such that

(i) $r(0)=0, r(1)=1$,

(ii) $r^{\prime}(t)>0$ on $0<t<1$,

(iii) $r^{\prime}(t)$ has a zero of multiplicity $N-1$ at $t=0$ and $t=1$.

8. Lemma. Suppose $E$ is connected, $[a, b] \subset E, u, v \in C^{N}(E-(a, b))$, $R \in C[a, b]$, and $C>0$. If $u$ and $v^{\prime}$ are positive at $t=a$ and $t=b$, $v(a)<v(b)$ and $R(t)>0$ on $(a, b)$, then there are extensions $U, V \in C^{N}(E)$ of $u, v$ respectively such that

(i) $U(t)>0$ on $a \leqq t \leqq b$,

(ii) $\int_{a}^{b} U(t) R(t) d t=C$,

(iii) $v^{\prime}(t)>0$ on $a \leqq t \leqq b$.

Proof. Let $P_{a}(t)$ and $P_{b}(t)$ denote the Lagrange interpolation polynomials which assume the initial values of $u(t)$ at $t=a$ and $t=b$, respectively. For all small $h>0, P_{a}(t)$ and all its derivatives are onesigned in $(a, a+h)$; a similar statement is true for $P_{b}(t)$.

Let $r(t)$ be as in Lemma 7 . For any constant $k>0$ all sufficiently small $h>0$ put

$$
\begin{aligned}
A_{h k}(t) & =u(t) \quad \text { if } t \notin(a, b), \\
& =r\left(\frac{t-a}{h}\right) k+\left[1-r\left(\frac{t-a}{h}\right)\right] P_{a}(t) \quad \text { if } t \in[a, a+h], \\
& =k \quad \text { if } t \in[a+h, b-h], \\
& =r\left(\frac{t-b+h}{h}\right) P_{b}(t)+\left[1-r\left(\frac{t-b+h}{h}\right)\right] k \\
& \text { if } t \in[b-h, b] .
\end{aligned}
$$

Then $A_{h k}(t)>0$ on $[a, b]$ for all small $h>0$ and $A_{h k} \in C^{N}(E)$. If $h>0$ is sufficiently small, then $\int_{a}^{b} A_{h k}(t) R(t) d t<C$ for all small $k>0$, and $\int_{a}^{b} A_{h k}(t) R(t) d t>C$ for all large $k>0$. This integral is a continuous function of $k$ for fixed $h$, and therefore (i) and (ii) hold with $U=A_{h k}$ for some pair $h, k$.

To establish (iii), apply the previous result to $v^{\prime}$ to obtain an extension $V_{1}$ such that $V_{1}>0$ on $[a, b]$ and $\int_{a}^{b} V_{1}(t) d t=v(b)-v(a)$. Define 
$V(t)=v(t)$ off $[a, b], V(t)=\int_{a}^{t} V_{1}(s) d s+v(a)$ on $a \leqq t \leqq b$. Then $V$ extends $v, V \in C^{N}(E)$, and $V^{\prime}>0$ on $[a, b]$.

9. Lemma. Suppose $[a, b]$ is contained in $T, T$ is connected, and $A, B, C$ are positive constants. Given $f$ and $g$ in $C^{N}(T-(a, b))$ such that $f^{2} g^{\prime}>0$ and $g(a)=2 k \pi, g(b)=2 k \pi+\pi$ for some integer $k \geqq 0$, there are extensions $F$ and $G$ of $f$ and $g$, respectively, such that $F$ and $G$ are in $C^{N}(T)$ and

(i) $F^{2} G^{\prime}>0$ on $[a, b]$,

(ii) $\int_{a}^{b} t^{p} F(t) \sin G(t) d t \geqq A, 0 \leqq p \leqq N-1$,

(iii) $\int_{a}^{b} F(t) \cos ^{+} G(t) d t=B, \int_{a}^{b} F(t) \cos ^{-} G(t) d t=C$.

Proof. If smoothness were not important, then on $a \leqq t \leqq b, F(t)$ could be taken to be a step function with three steps, and $G(t)$ a piecewise linear function of three segments. These functions can be modified via Lemma 8 to yield the required functions of class $C^{N}$. The central idea in establishing (ii) and (iii) is to select an interval $[c, d]$ inside $(a, b)$ and make $F(t)$ constant and $G(t)$ linear on $[c, d]$, with $G(d)=2 k \pi+\pi / 2$. Then (ii) is obtained by choosing $F(t)$ large and $G^{\prime}(t)$ small on $[c, d]$. Since (ii) remains true if $G^{\prime}(t)$ is decreased on $[c, d]$, the integral $\int_{c}^{d} F(t) \cos G(t) d t$ can be made smaller than the given constant $B$. Now $G(t)$ is defined via Lemma 8 on the remainder of $[a, b]$, subject to the conditions $G \in C^{N}$ and $G^{\prime}>0$. Then $F(t)$ is defined on $[a, c]$ and $[d, b]$ in order to satisfy $\int_{a}^{b} F(t) \cos ^{+} G(t) d t=B$, $\int_{a}^{b} F(t) \cos ^{-} G(t) d t=C$, and $F(t)>0$ (see Lemma 8), demonstrating (i)-(iii).

10. ThEOREM. Given an integer $N>2$, there exists an Nth order linear differential equation $\mathrm{Lz}=0$ such that

(i) $L z=0$ is nonoscillatory on $t \geqq 0$,

(ii) $L^{*} w=0$ is strongly oscillatory on $t \geqq 0$,

(iii) $\eta_{p}(t)$ exists for all $t \geqq 0$, all integers $p \geqq 1$.

Proof. The equation $L z=0$ will have the special form $\left[\left(p(t) z^{\prime}\right)^{\prime}+q(t) z\right]^{(\nu)}=0$, where $N=\nu+2$; see Lemma 3. To construct the second order equation, let $J_{n}=\left[a_{n}, b_{n}\right], n \geqq 1$, be an interval in $[0, \infty)$, and suppose that $b_{1}=0, b_{n}<a_{n+1}, a_{n} \rightarrow \infty$, and $\int_{J}\left|t^{N-3}\right| d t$ is finite, where $J$ denotes the union of all the $J_{n}$ 's, $n=1,2,3, \cdots$.

Define a step function $f$ and a linear function $g$ on $J$ by the following scheme: $g\left(a_{n}\right)$ is an odd multiple of $\pi, g\left(b_{n}\right)$ is an even multiple of $\pi, g\left(a_{n}\right)$ is less than $g\left(b_{n}\right), g\left(a_{n+1}\right)=g\left(b_{n}\right)+\pi, x_{n} \in(0,1)$ is chosen so that $w^{\prime \prime}+x_{n}^{-2} w=0$ has a nontrivial solution with $n+1$ zeros on $J_{n}, g(t)=x_{n}^{-1}\left(t-a_{n}\right)+g\left(a_{n}\right)$ on the interval $J_{n}$, and $f(t)=x_{n}$ on $J_{n}$, $n=1,2,3, \cdots$.

For such $f$ and $g$, the integrals $\int_{J}\left|t^{p} f(t) \sin g(t)\right| d t, 0 \leqq p \leqq N-3$, 
$\int_{J} f(t)|\sin g(t)| d t, \int_{J} f(t)|\cos g(t)| d t$ are finite, and each is bounded by the integral $\int_{J} \max \left\{\left|t^{p}\right|: 0 \leqq p \leqq N-3\right\} d t$.

Define $I_{n}=\int_{J_{n}} f(t)|\cos g(t)| d t, \quad n \geqq 1$, and put $B_{1}=I_{1}+I_{2}+I_{3}$, $B_{n}=I_{n+2}$ for $n>1, C_{n}=I_{n}$ for $n \geqq 1$. Let $T=\left[a_{n}, b_{n+1}\right], A=n, B=B_{n}$, $C=C_{n}$ in Lemma 8 to extend $f$ and $g$ as $C^{N}$ functions to the interval between $J_{n}$ and $J_{n+1}, n \geqq 1$. The extended functions will be denoted by $f$ and $g$ also.

Since $\sum_{n=1}^{k-1}\left(B_{n}-C_{n}\right)>\int_{J_{k}} f(t)|\cos g(t)| d t(k>1), \sum_{n=1}^{\infty} B_{n}, \sum_{n=1}^{\infty} C_{n}$ both equal $\int_{J} f(t)|\cos g(t)| d t$, and $\int_{I_{n}} f(t) \cos g(t) d t=0$, it can be verified that $\int_{0}^{t} f(t) \cos g(t) d t$ is positive for $t>0$, and

$$
\lim _{t \rightarrow \infty} \int_{0}^{t} f(t) \cos g(t) d t=0 .
$$

The construction also leads to

$$
\int_{0}^{\infty} t^{p} f(t) \sin ^{+} g(t) d t=\infty, \quad \int_{0}^{\infty} t^{p} f(t) \sin ^{-} g(t) d t<\infty, 0 \leqq p \leqq N-3 .
$$

Put $x(t)=f(t) \cos g(t), y(t)=f(t) \sin g(t)$. By Lemma 2, $x(t)$ and $y(t)$ form a fundamental set of solutions for a second order equation of the form

$$
\left(p(t) z^{\prime}\right)^{\prime}+q(t) z=0,
$$

where $p(t)=1 /\left(f^{2}(t) g^{\prime}(t)\right)$. The preceding paragraph shows that $x(t)$ and $y(t)$ satisfy Lemma 5 .

By Lemma $5, L z=0$ and $L^{*} w=0$ are strongly oscillatory and nonoscillatory, respectively. Lemma 6 shows that $\eta_{p}(t)$ exists for all $t \geqq 0$, all integers $p \geqq 1$. This proves Theorem 10 and answers the four questions in the Introduction in the negative for $n>2$.

\section{REFERENCES}

1. J. M. Dolan, Oscillatory behavior of solutions of linear ordinary differential equations of third order, Ph.D. Dissertation, University of Tennessee, Knoxville, Tenn., 1967.

2. - - On the relationship between the oscillatory behavior of a linear third-order equation and its adjoint, J. Differential Equations (to appear).

3. G. B. Gustafson, Conjugate point properties for nth order linear differential equations, Ph.D. Dissertation, Arizona State University, Tempe, Ariz., 1968.

4. M. Hanan, Oscillation criteria for third-order linear differential equations, Pacific J. Math. 11 (1961), 919-944. MR 26 \#2695.

5. P. Hartman, Ordinary differential equations, Wiley, New York, 1964. MR 30 \#1270.

University of Utah, Salt Lake City, Utah 84112 